\title{
The Effects of Clumping and Substructure on ICM Mass Measurements
}

\author{
B. Mathiesen, A. E. Evrard \\ Dept. of Physics, University of Michigan, Ann Arbor, MI 48109 \\ J. J. Mohr"] \\ Dept. of Astronomy and Astrophysics, University of Chicago, Chicago, IL 60637
}

\begin{abstract}
We examine an ensemble of 48 simulated clusters to determine the effects of small-scale density fluctuations and large-scale substructure on X-ray measurements of the intracluster medium (ICM) mass. We measure RMS density fluctuations in the ICM which can be characterized by a mean mass-weighted clumping factor $C \equiv\left\langle\rho^{2}\right\rangle /\langle\rho\rangle^{2}$ between 1.3 and 1.4 within a density contrast of 500. These fluctuations arise from the cluster history of accretion shocks and major mergers, and their presence enhances the cluster's luminosity relative to the smooth case. We expect, therefore, that ICM mass measurements utilizing models which assume uniform density at a given radius carry a bias of order $\sqrt{C} \approx 1.16$. We verify this result by performing ICM mass measurements on $\mathrm{X}$-ray images of the simulations and finding the expected level of bias.

The varied cluster morphologies in our ensemble also allow us to investigate the effects of departures from spherical symmetry on our measurements. We find that the presence of large-scale substructure does not further bias the resulting gas mass unless it is pronounced enough to produce a second peak in the image of at least $1 \%$ the maximum surface brightness. We analyze the subset of images with no secondary peaks and find a bias of $9 \%$ and a Gaussian random error of $4 \%$ in the derived mass.
\end{abstract}

Subject headings: galaxies: clusters: general — intergalactic medium

\footnotetext{
${ }^{1}$ Chandra Fellow
} 


\section{Introduction}

Studies of the intracluster medium (ICM) address many important cosmological and astrophysical questions. Precision measurements of individual ICM spectra and metallicities can be used to constrain models of massive star formation. The slope and evolution of cluster scaling relations (e.g. the luminosity vs. temperature or ICM mass vs. temperature) can provide insight into the nature and importance of galactic winds, as well as the background cosmology in which the ICM develops. In addition, current models of structure formation suggest that the mass components of clusters are representative of the universe as a whole; for this reason there has been much effort expended towards measuring the contributions of dark matter, ICM, and galaxies in the population. The mean ICM mass fraction is therefore a useful lower limit on the global baryon fraction, and can also be used to constrain $\Omega_{0}$ if combined with primordial nucleosynthesis calculations.

Many studies have been carried out recently which attempt to measure the form of the ICM density profile using X-ray images (e.g. Mohr, Mathiesen, \& Evrard 1999 (MME); White, Jones, \& Forman 1997; Loewenstein \& Mushotzky 1996; David, Jones, \& Forman 1995, White \& Fabian 1995). Following the lead of early work in the field (Forman \& Jones 1982), such studies typically create an analytic model for the azimuthally averaged ICM density profile, and use the $\mathrm{X}$-ray surface brightness to constrain its parameters. These models generally assume that the ICM is uniform at a given radius, that the density profile decreases monotonically from the center, and that the cluster is spherically symmetric.

These assumptions break down in real clusters; even apparently relaxed clusters tend to exhibit small asphericities. The prevalence of accretion events and major mergers in the local population is also well established, which calls into question the validity of a monotonic density profile. The assumption of a uniform ICM is a reasonable approximation, but it too must fail on sufficiently small scales. A certain level of random density fluctuations is to be expected, and even minor accretion events are found in simulations to produce persistent mild shocks and acoustic disturbances in the ICM. The small scatter in observed scaling relations, for example the ICM mass and mass fraction vs. temperature (MME), implies that these assumptions produce results with at least $20 \%$ precision; this fact inspires confidence. It is important, however, to directly measure the systematic errors incurred by these inaccuracies.

In accordance with the increasing quality of $\mathrm{X}$-ray observations, the scientific community has recently begun to address these issues. A number of groups are investigating the possibility of a multiphase medium (Thomas 1998, Lima Neto et al. 1997, Waxman \& Miralde-Escude 1995), although most of the work to date focuses on cooling flows rather than global ICM properties. A notable exception is the work of Gunn \& Thomas (1996), 
who examine the effect of a multiphase ICM on baryon fraction measurements. There have also been attempts to get around the assumption of spherical symmetry by using the isophotal area (Mohr \& Evrard 1997) or elliptical isophotes (Knopp \& Henry 1996, Buote \& Canizares 1996) to constrain a density model. All of these methods, however, still fall prey to one or more of the assumptions mentioned above.

Hydrodynamic simulations of cluster evolution are ideally suited to the task of estimating the systematic errors incurred by these assumptions. In this study, we produce realistic X-ray surface brightness images from a set of simulations and measure ICM masses by fitting the emission profile to a spherically symmetric beta model for the density. Our independent knowledge of the three-dimensional structure allows us to observe the effects of gross substructure and small-scale density fluctuations on the derived ICM mass directly, and correlate these errors with the kind of substructure present. In $\S 2$ of this paper, we present some detail on the nature of the simulations and how we create our X-ray images. In $\S 3$, we describe how we analyze these images and extract ICM density profiles. In $\S 4$, we compare the "observed" masses to the simulations and correlate the errors with structural properties of the ICM. Finally, in $\S 5$ we restate our results and remark on how they can be applied to real observations. Our results are phrased throughout in a manner indpendent of the Hubble constant $H_{0}$.

\section{Data}

We use an ensemble of 48 hydrodynamical cluster simulations, divided among four different cold dark matter (CDM) cosmological models. These models are (i) SCDM $\left(\Omega_{0}=1, \sigma_{8}=0.6, h=0.5, \Gamma=0.5\right)$; (ii) $\tau \mathrm{CDM}\left(\Omega_{0}=1, \sigma_{8}=0.6, h=0.5, \Gamma=0.24\right)$; (iii) $\operatorname{OCDM}\left(\Omega_{0}=0.3, \sigma_{8}=1.0, h=0.8, \Gamma=0.24\right)$; and (iv) $\Lambda \operatorname{CDM}\left(\Omega_{0}=0.3, \lambda_{0}=0.7\right.$, $\left.\sigma_{8}=1.0, h=0.8, \Gamma=0.24\right)$. Here the Hubble constant is $100 h \mathrm{~km} \mathrm{~s}^{-1} \mathrm{Mpc}^{-1}$, and $\sigma_{8}$ is the power spectrum normalization on $8 h^{-1}$ Mpc scales. The initial conditions are Gaussian random fields consistent with a CDM transfer function with the specified $\Gamma$ (Davis et al. 1985). The baryon density is set in each case to a fixed fraction of the total density $\left(\Omega_{b}=0.2 \Omega_{0}\right)$. The simulation scheme is $\mathrm{P} 3 \mathrm{MSPH}$; first a $\mathrm{P}^{3} \mathrm{M}$ (DM only) simulation is used to find cluster formation sites in a large volume, then a hydrodynamic simulation is performed on individual clusters to resolve their DM halo and ICM structure in detail. The resulting cluster sample covers a little more than a decade in total mass, ranging from about $10^{14}$ to $2 \times 10^{15} M_{\odot}$.

We work with $\mathrm{X}$-ray surface brightness maps derived from the simulations according to procedures described by Evrard (1990a,b). The particles representing pieces of the ICM are 
treated as bremsstrahlung emitters at the local temperature and density, and this emission is distributed over the image pixels according to a two-dimensional Gaussian with a width equal to that of the particle's SPH smoothing kernel. Emission is collected in the energy band $[0.1,2.4] \mathrm{keV}$, and the resulting surface brightness in each pixel is converted to counts per second according to an approximate ROSAT PSPC energy conversion factor. We then "observe" this model image using an effective area map of the PSPC and an exposure sufficient to yield $10^{4}$ cluster photons, comparable to ROSAT exposures of clusters in the Edge sample (Edge et al. 1990). Finally, each pixel is given a Poisson uncertainty based on the number of photons and the whole image is smoothed on a scale of $24.4^{\prime \prime}$ to improve the signal-to-noise ratio. Angular distances are calculated as $d_{A}=c z / H_{0}$ with $z=0.06$, so the physical smoothing scale is either 42.6 or $26.6 \mathrm{kpc}$. Note that beause we are trying to isolate the systematic errors due to substructure, we do not add Poisson noise to the image; the error bars are only used to assign appropriate weights to the data in the analysis.

It should be noted that the simulations do not include certain processes known to be present in real clusters, such as radiative cooling and the injection of gas and energy by galactic supernovae. The cooling time for at least $99 \%$ of the SPH particles is much longer than the Hubble time, and would have little effect on the structure of the ICM; nevertheless, our simulations cannot develop cooling flows. Detailed studies of real cooling flows find mass deposition rates no larger than hundreds of solar masses per year, suggesting that they comprise only a small fraction of the ICM mass (White et al. 1998). Simulations which include galactic winds create clusters with more realistic density profiles, but the winds are not found to greatly affect the temperature structure of the gas (Metzler \& Evrard 1994). It is conceivable that the presence of galactic winds would also create more clumping in the ICM, but this effect is probably negligible next to the variations caused by accretion events.

The clusters display great morphological diversity, ranging from examples which appear almost perfectly spherical and relaxed to others which are undergoing a three-way merger event. Each cluster is imaged in three orthogonal projections, allowing us to compare the biases caused by substructure along the line-of-sight and substructure in the plane of the sky. We use all three projections in general, but confine ourselves to one projection per cluster when making statistical comparisons to insure that the probabilities are derived from statistically independent data. Further details on the nature of these simulations can be found in both MME and Mohr \& Evrard (1997). 


\section{Image Analysis}

We fit the emission to a beta model, with three-dimensional density profile $\rho(r)=\rho_{0}\left[1+\left(r / r_{c}\right)^{2}\right]^{-3 \beta / 2}$ (Cavaliere \& Fusco-Femiano, 1978). In this model $r_{c}$ and $\beta$ are free parameters to be constrained by the $\mathrm{X}$-ray emission profile, and $\rho_{0}$ is found by normalizing the emission integral to the bolometric luminosity of the cluster.

Producing a cluster emission profile appropriate for fitting requires three steps. First, the emission center is found by sliding a circular aperture of radius 10 pixels over the image and minimizing the distance between the center of the aperture and the centroid of the X-ray photon distribution within the aperture. This approach converges even in cases where the cluster emission is significantly skew (Mohr, Fabricant, \& Geller 1993). Second, we calculate the azimuthally averaged radial profile. Finally, each point in the profile is assigned a Poisson uncertainty based on the number of photons in the annulus; this allows the fitting program to assign approprate relative weights to the datapoints.

The PSPC point spread function (PSF) reduces a cluster's central intensity and increases the apparent value of $r_{c}$. This effect changes the best-fit values of our model parameters and biases the derived ICM mass if left untreated. The incurred error is potentially significant for clusters with small angular diameter or pronounced cooling flows, and is simulated here by the image smoothing mentioned in $\S 2$. Thus, rather than fitting the beta model directly to our profile, we first convolve it with a PSF appropriate for our

smoothing kernel. Details on the mathematics of this technique can be found in MME and Saglia et al. (1993).

The fitting was performed from the center of the cluster out to $r_{500}$, the radius at which the mean interior density is 500 times the critical density. This measure was chosen to probe the easily visible region of each cluster given typical backgrounds and sensitivities, as well as for physical reasons which will become apparent in the next section. By fitting out to a fixed fraction of the virial radius rather than a fixed metric radius, we insure that we are probing regions with similar dynamical gravitational time scales. The ratio $\Upsilon$ between cluster baryon fractions and the universal baryon fraction has also been calibrated by simulations; a bias factor of $\Upsilon=0.9 \pm 0.1$ was found at $r_{500}$ (Evrard 1997). Recent work with an independent simulation has corroborated this result, finding $\Upsilon=0.92 \pm 0.06$ at $r_{200}$ (Frenk et al. 1999). The radius $r_{500}$ is found in our simulations to scale as $1.4 h^{-1}(T / 10 \mathrm{keV})^{1 / 2} \mathrm{Mpc}$, regardless of cosmology.

Finally, we checked our process by fitting 100 monte carlo images of true beta models with similar smoothing and individual realizations of Poisson noise, and found a chi-squared distribution consistent with a perfect match between the fitting function and the underlying 
model. This confirms that the high chi-squared values obtained in some images result from real physical deviations from a spherical beta model. A more complete discussion of our method, as applied to real PSPC data, is given in MME.

\section{Results}

We measure the variance of density fluctuations in spherical shells around the ICM particle with the lowest gravitational potential. This variance is expressed in terms of a clumping factor $C$,

$$
C \equiv\left\langle\rho^{2}\right\rangle /\langle\rho\rangle^{2} .
$$

Each ICM particle in the simulation has mass $m_{\text {gas }}$. For a given shell lying between radii $r_{1}$ and $r_{2}$ with $N_{12}$ gas particles, $\langle\rho\rangle$ is defined as the total mass in gas particles divided by the volume of the shell, $m_{\text {gas }} N_{12} / V$. The numerator is calculated as

$$
\left\langle\rho^{2}\right\rangle \equiv \frac{1}{V_{12}} \int_{r_{1}}^{r_{2}} d^{3} r \rho^{2} \rightarrow \frac{m_{\text {gas }}}{V_{12}} \sum_{i}^{N_{12}} \rho_{i},
$$

with the right-hand term being the Lagrangian limit of the integral and $\rho_{i}$ the SPH gas density of particle $i$ (Evrard 1988).

The results of this analysis are displayed in Figure 1. Although more complicated structure is visible in some of our clusters, the average magnitude of density fluctuations interior to $r_{500}$ seems to be a slowly increasing function of radius. The radial bins are chosen such that they represent fixed logarithmic intervals in the density constrast $\delta_{c}$. Dramatic increases in clumping are occasionally seen between $r_{500}$ and the more traditional virial radius; this is another reason that for choosing to work within a density constrast of 500 . Extending our analysis to the virial radius would return essentially the same results with lower statistical significance.

The presence of these fluctuations enhances the cluster luminosity over what would be expected for a smooth, single-phase ICM. The emissivity in a given shell is given by

$$
\varepsilon(r)=r^{2} d r \int d \Omega \frac{\rho^{2}(r, \Omega)}{\mu_{e} \mu_{H} m_{p}^{2}} \Lambda(T, \Omega) .
$$

If the density fluctuations are modest, the temperature and ionization structure of the gas in a given shell will be approximately uniform. Taking these factors outside the integral and using equation (1) to incorporate our clumping factor, we have

$$
\varepsilon(r)=4 \pi r^{2} d r \frac{\Lambda(T)}{\mu_{e} \mu_{H} m_{p}^{2}}\langle\rho(r)\rangle^{2} C(r) .
$$


$C$, therefore, represents the factor by which a shell's emissivity is enhanced over the single-phase case. This formalism can be invoked to describe a true multiphase medium under arbitrary constraints by choosing an appropriate function $C(r)$. Nagai, Evrard, \& Sulkanen (1999) look at such distributions in more detail, investigating the observable consequences of applying isobaric multiphase models.

For each cluster we calculate the mass-weighted mean $\bar{C}$ over all shells as an approximation to the overall bias on its total luminosity and inferred core density $\rho_{0}^{2}$. These values, averaged again over all clusters within a cosmological model, are as follows: $\bar{C}_{o}=1.40, \bar{C}_{s}=1.32, \bar{C}_{\Lambda}=1.29$, and $\bar{C}_{\tau}=1.38$. The mean values of $M_{\beta} / M_{\text {true }}$ for the four cosmologies are $1.34(\mathrm{OCDM}), 1.13(\mathrm{SCDM}), 1.11(\Lambda \mathrm{CDM})$, and $1.14(\tau \mathrm{CDM})$. The mass error distributions for the four cosmologies are mutually consistent, so we combine them to determine a mean ICM bias for the entire ensemble of $1.18 \pm 0.02$. We also calculate the ensemble mean of $\bar{C}$, arriving at $1.34 \pm 0.02$. Since the total luminosity is proportional to the square of the central gas density, we expect an overall bias in our ICM mass estimates of $\bar{C}^{1 / 2}$, or $1.16 \pm 0.01$. The error bars given here are one standard deviation of the mean, so this is excellent agreement. We do not, however, find a strong correspondence between the level of clumping and the ICM mass error in individual clusters, however. Variations in the shape of the mass profile and large-scale asymmetries, which contribute a random component to the error, mask this relationship.

It is difficult to judge what weighting scheme is most appropriate for this calculation, since the pivotal regions of the emission profile vary from cluster to cluster. The central points are very accurate and do much to constrain the fit, but are few in number; the outer regions have large error bars but many more data points. We began our anaysis with a mass-weighted clumping factor under the simple reasoning that it would greatly favor neither region. We also performed the exercise of weighting the shells by their luminosity (which gives more importance to the core) and volume (which emphasizes the outskirts). The resulting ensemble mean clumping factors do not vary greatly under the different weighting schemes: we found averages of $\bar{C}=1.27$ for luminosity-weighted shells and $\bar{C}=1.39$ for volume-weighted shells.

We also attempt to identify correlations of mass error with large-scale substructure signatures. A surprising result is that we find no correlation of $M_{\beta} / M_{\text {true }}$ with centroid shift, which has been found to be a good indicator of a cluster's dynamical state (Mohr, Evrard, Fabricant, \& Geller 1995). Even those clusters with the highest centroid shifts don't show a significantly different gas mass bias from the rest of the sample. Apparently, azimuthally averaging the cluster profile compensates for mild asphericities very well. We also looked for correlations of mass error with various bulk properties of the ICM such as 
mass, emission-weighted temperature, and luminosity, finding none. There is evidence for a weak correlation of mass error with the model parameters $\beta$ and $r_{c}$, but this can be entirely attributed to the tendency for strongly bimodal clusters to have profiles which are not well fit by the model. Although our clusters typically have steeper profiles than are observed in reality (c.f.), the lack of shape dependence indicates that this is probably not a problem. In none of the above tests were there discernible differences among the four cosmologies.

In fact, we found only one ICM property to be correlated with $\delta M$. Defining a "secondary peak" as a local maximum in the surface brightness at least $1 \%$ of the global maximum, we find that the most important property is the existence of secondary peaks within $r_{500}$. We attempted to further quantify the degree of asymmetry caused by subclumping, but the mass error turned out to be uncorrelated with the number and strength of the subpeaks. We therefore divide our ensemble into two subsets on this basis, hereafter referred to as "regular" and "bimodal" clusters for ease of language. Note that the subset of "regular" clusters contains examples with large centroid shifts or asymmetries, and the "bimodal" subset includes a few trimodal clusters as well. The two subsets comprise $62 \%$ and $38 \%$ of the total, respectively. Figure 2 displays a histogram of ICM mass errors for all the images in our ensemble. The shaded region corresponds to the regular clusters, while the remaining subset represents the bimodal sample.

The bimodal population has a distribution of mass errors with mean $\delta M=0.188$ $\left(\delta M=1-M_{\beta} / M_{\text {true }}\right)$ and standard deviation $\sigma=0.084$. The remaining population has a distribution with $\delta \bar{M}=0.093$ and $\sigma=0.041$. Applying the $\mathrm{K}-\mathrm{S}$ test, we find that the two populations are drawn from different distributions with very high confidence (greater than $5 \sigma$ ) and that the subset of non-bimodal images has an error distribution consistent with the Gaussian form. In applying the $\mathrm{K}-\mathrm{S}$ tests we worked with a subset of the data consisting of just one image from each simulation, in order to maintain statistical independence. The means and standard deviations just quoted, however, reflect the distribution of the entire image ensemble. The smaller samples in each category have distributions entirely consistent with the complete sample, but we feel that the stated values better reflect the size of real uncertainties by taking advantage of multiple projections.

Within our sample there are also 17 (out of 144) images which contain a major subclump in line-of-sight with the primary cluster and indistinguishable as a secondary peak. (A major subclump is one which produces a secondary peak when the cluster is viewed from a different angle.) These images, which occur in both the regular and bimodal populations, have a distribution in $\delta M$ consistent with the remaining set of bimodal images. This is reasonable given the similarity of their three-dimensional physical structures. Their partial membership in the set of regular images, however, does not change the shape or 
mean of that distribution significantly. Such occurences seem to be sufficiently uncommon that our heuristic classification system remains useful. Since strongly bimodal clusters are more uncommon than our simulations indicate, real samples will probably suffer even less from this contamination.

\section{Conclusions}

Assuming a density profile which is uniform at a given radius introduces a significant bias into measurements of the ICM mass, because it ignores the presence of luminosity enhancements from overdense regions. Our simulations indicate that these small-scale fluctuations produce a mean overestimate of $\sim 10 \%$ when we confine our analysis to regular clusters. The advent of spatially resolved X-ray spectral imaging should allow us to test for irregularity in ICM structures and constrain the level of these fluctuations with direct observations; in the meantime it seems prudent to apply a correction of this scale to current measurements, as is done in MME. The relationship between these fluctuations and the cluster accretion history remains an open question, but this analysis suggests that there is no strong connection.

Applying our spherically symmetric model to a cluster's azimuthally averaged surface brightness profile returns reasonably accurate ICM mass measurements even in clusters exhibiting significant asymmetries, so long as there are no significant secondary peaks in the image. The assumption of spherical symmetry, while formally invalid in most cases, seems to be borne out in practice in that the process of azimuthal averaging returns a mean density profile which is unbiased by the presence of moderate substructure. Such deviations from spherical symmetry contribute a random error of $\sim 5 \%$ to ICM mass measurments under our method. The subset of bimodal (or multi-peaked) clusters has a much larger bias and dispersion, and is perhaps best excluded from population studies of the ICM.

This research was supported by NASA grants NAG5-2790, NAG5-3401, and NAG57108, as well as NSF grant AST-9803199. JJM is supported through Chandra Fellowship grant PF8-1003, awarded through the Chandra Science Center. The Chandra Science Center is operated by the Smithsonian Astrophysical Observatory for NASA under contract NAS8-39073.

\section{REFERENCES}


Buote D. A. \& Canizares C. R., 1996, ApJ, 457, 565

Cavaliere A. \& Fusco-Femiano R., 1978, A\&A, 70, 677

David L. P., Jones C., \& Forman W., 1995, ApJ, 445, 578

Davis M., Efstathiou G., Frenk C. S., \& White S. D. M., 1985, ApJ, 292, 371

Edge A. C., Stewart G. C., Fabian A. C., \& Arnaud K. A., 1990, MNRAS, 245, 559

Evrard A. E., 1988, MNRAS, 235, 911

Evrard A. E., 1990a, ApJ, 363, 349

Evrard A. E., 1990b, in Oegerle W. R., Fitchett M. J., Danly L., eds, Clusters of Galaxies, Cambridge Univ. Press, Cambridge, 287

Evrard A. E., 1997, MNRAS, 292, 289

Fabian A. C., Hu E. M., Cowie L. L., Grindlay J., 1981, ApJ, 248, 47

Forman W. \& Jones C., 1982, ARA\&A, 20, 547

Frenk C. S. et al., 1999, ApJ, submitted

Gunn K. F. \& Thomas P. A., 1996, MNRAS, 281, 1133

Knopp G. P. \& Henry J. P., 1996, ApJ, 472, 125

Loewenstein M. \& Mushotzky R. F., 1996, ApJ, 471, 83

Metzler C. A. \& Evrard A. E., 1994, ApJ, 437, 564

Mohr J. J. \& Evrard A. E., 1997, ApJ, 491, 38

Mohr J. J., Evrard A. E., Fabricant D. G., \& Geller M. J., 1995, ApJ, 447, 8

Mohr J. J., Fabricant D. G., \& Geller M. J., 1993, ApJ, 413, 492

Mohr J. J., Mathiesen B., \& Evrard A. E., 1999, ApJ, in press, astro-ph/9901281

Nagai D., Evrard A. E., \& Sulkanen M. E., 1999, submitted to MNRAS(astro-ph/9903308)

Lima Neto G. B., Pislar V., Durret F., Gerbal D. \& Slezak, E., 1997, A\&A, 327, 81

Saglia R. P., Bertschinger E., Baggley G., Burstein D., Colless M., Davies R. L., McMahan R. K., \& Wegner G., 1993, MNRAS, 264, 961 
Thomas P. A., 1998, MNRAS, 299, 349

Waxman, E. \& Miralda-Escude J., 1995, ApJ, 451, 451

White D. A. \& Fabian A. C., 1995, MNRAS, 273, 72

White D. A., Jones C., \& Forman W., 1997, MNRAS, 292, 419 
Fig. 1.- The rms density fluctuations in spherical shells for all 48 simulated clusters. The magnitude of these fluctuations does not appear to depend significantly on the cosmological background and is reasonably stable within $r_{500}$. The dotted line represents the massweighted mean $\bar{C}$, averaged over all clusters in that cosmology.

Fig. 2.- The distribution of systematic errors in ICM mass measurements based on the beta model. The full histogram is for the entire image ensemble (three per cluster); the shaded region is for the subset of regular cluster images. The curve is a Gaussian distribution with the same mean and standard deviation as the shaded histogram. There is an outlier at $M_{\beta} / M_{\text {true }}=1.8$ which lies beyond the limits of the plot. 


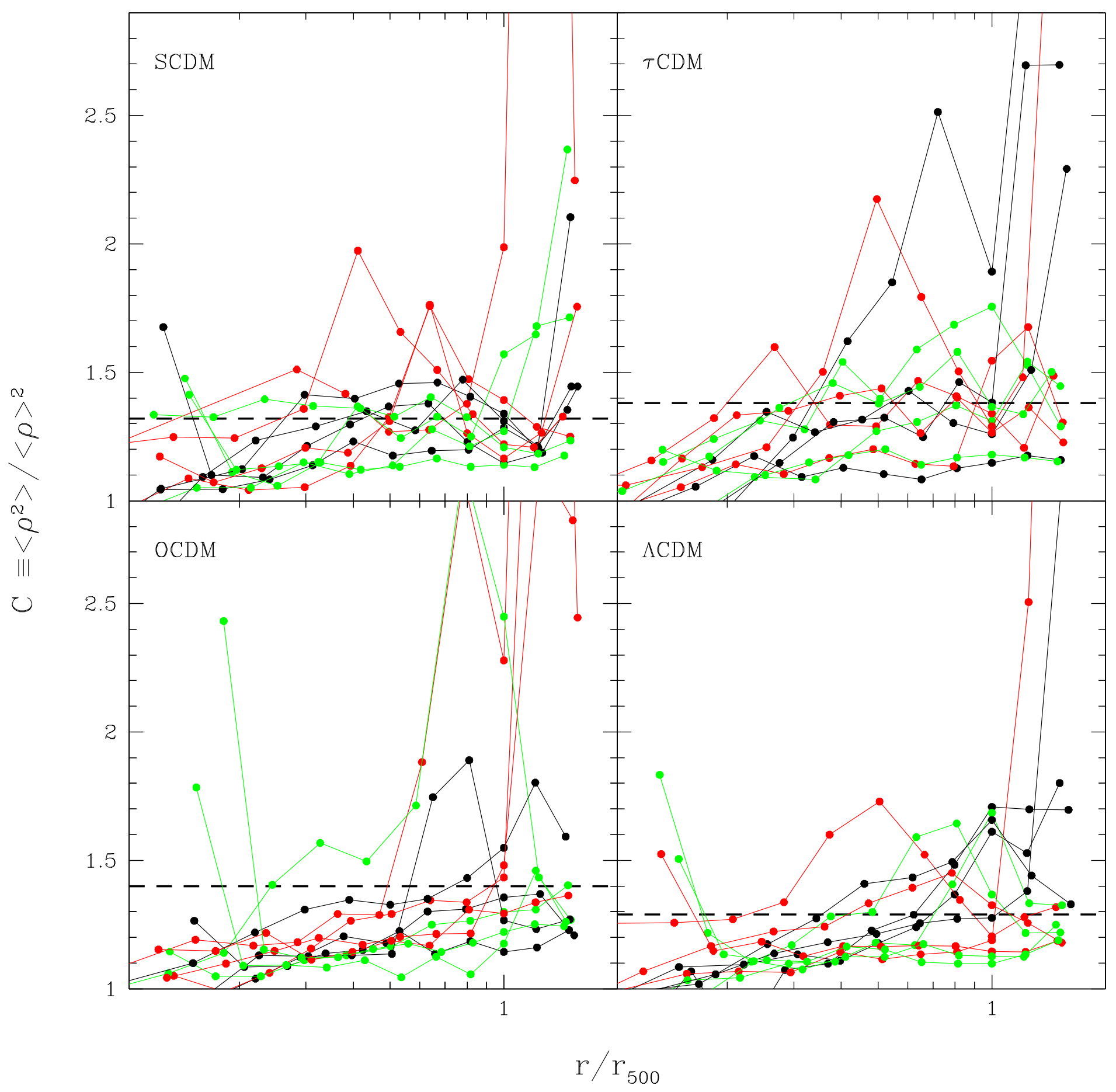




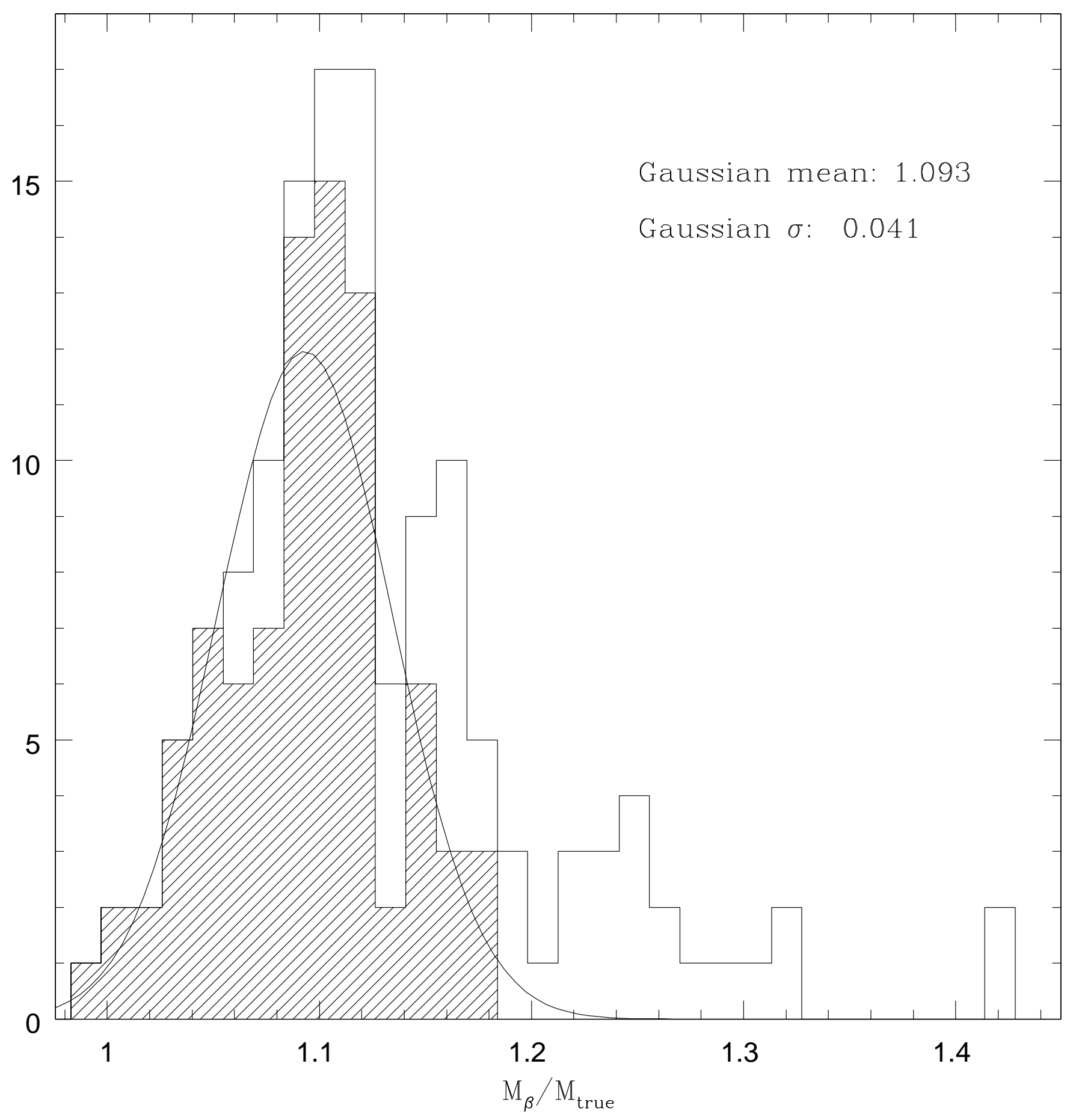

